\title{
COSFIRE: A Brain-Inspired Approach to Visual Pattern Recognition
}

\author{
George Azzopardi $\left.{ }^{(}\right)$and Nicolai Petkov \\ Johann Bernoulli Institute for Mathematics and Computer Science, \\ University of Groningen, Groningen, The Netherlands \\ $\{$ g.azzopardi, n. petkov\}@rug.nl
}

\begin{abstract}
The primate visual system has an impressive ability to generalize and to discriminate between numerous objects and it is robust to many geometrical transformations as well as lighting conditions. The study of the visual system has been an active reasearch field in neuropysiology for more than half a century. The construction of computational models of visual neurons can help us gain insight in the processing of information in visual cortex which we can use to provide more robust solutions to computer vision applications. Here, we demonstrate how inspiration from the functions of shape-selective V4 neurons can be used to design trainable filters for visual pattern recognition. We call this approach COSFIRE, which stands for Combination of Shifted Filter Responses. We illustrate how a COSFIRE filter can be configured to be selective for the spatial arrangement of lines and/or edges that form the shape of a given prototype pattern. Finally, we demonstrate the effectiveness of the COSFIRE approach in three applications: the detection of vascular bifurcations in retinal fundus images, the localization and recognition of traffic signs in complex scenes and the recognition of handwritten digits. This work is a further step in understanding how visual information is processed in the brain and how information on pixel intensities is converted into information about objects. We demonstrate how this understanding can be used for the design of effective computer vision algorithms.
\end{abstract}

Keywords: Computational models of vision - COSFIRE - Trainable filters $\cdot$ Feature detection $\cdot$ Shape $\cdot$ Handwritten digits $\cdot$ Retinal fundus images $\cdot$ Traffic signs

\section{Introduction}

"If our perception of a certain line or curve depends on simple or complex cells, it presumably depends on a whole set of them, and how the information from such sets of cells is assembled at subsequent stages in the path to build up what we call percept of lines or curves (if indeed anything like that happens at all) is still a complete mystery." writes D.H. Hubel in his Nobel Price lecture [26]. 
In the following, we propose a way how to assemble the information from Gabor filters, that are mathematical models of simple and complex cells, in order to construct detectors of more complex stimuli, such as lines, angles, curves, line bifurcations and, more generally, local combinations of line and curve segments. We also demonstrate the effectiveness of these detectors in practical applications.

The brain processes visual information in the so called visual pathway. It consists of two parts, namely the ventral and the dorsal streams, that are responsible for, roughly speaking, 'what' and 'where' aspects. We are concerned with how the ventral stream processes visual information, which takes an input signal from the retina and transforms it into meaningful object representation. This stream comprises cortical areas V1, V2, V4, TE and TEO [19,52].

Simple and complex cells referred to above are found in areas V1 and V2 $[27,28]$. The understanding of their properties have been the focus of numerous electrophysiological studies $[2,14,15,35,51,53]$. Later, computational models were developed aiming at computer simulations of the function of these neurons $[1,3,33,47]$. These computational models gave the basis for biologically motivated contour detection algorithms in digital image processing. In particular, two-dimensional Gabor functions were proposed for computational modelling of these cells $[11,30]$. Gabor functions were then widely applied in diverse computer vision tasks, including edge detection [32,37], texture analysis $[9,17,23,29,49,50]$, image coding and compression [12], person identification based on iris pattern analysis [13], image enhancement [10], face recognition [36], motion analysis [42], and retrieval from image databases [54]. Further refinements of these models, include non-classical receptive field inhibition [43], also called surround suppression, and the filters that deploy this mechanism were shown to be effective detectors of object contours [21,22].

In contrast to areas $\mathrm{V} 1 / \mathrm{V} 2$, there is still little knowledge on how visual information is processed further in subsequent areas of the ventral pathway. Area V4 receives input from V1/V2 and is known to comprise neurons selective for various aspects of visual information, such as shape [38], color [55] or texture [24]. In this paper we are concerned with shape and, therefore, we are mainly interested in the function of shape-selective V4 neurons. An account of the properties of this type of neuron was given by Pasupathy and Connor [38]. They investigated the activations of such neurons in macaque monkeys, using a systematically designed data set of relatively simple contour features similar to those illustrated by Fig. 1 . They found that most (91\% of the 152) V4 neurons they studied were highly selective to curved contour features rather than to simple edges or bars. They also observed that V4 neurons are selective for the orientation of the contour feature, i.e. these neurons exhibited strong responses to angles and curves pointing in a specific direction. However, such a neuron may also be activated (with less than the maximum response) by stimuli differing slightly in orientation and/or curvature. Further analysis on V4 neurons was performed on a more complex data set including closed contour stimuli containing a combination of convex and concave contour elements [39]. The results of that study have shown that some V4 neurons are sensitive to a single convex or concave contour element, 


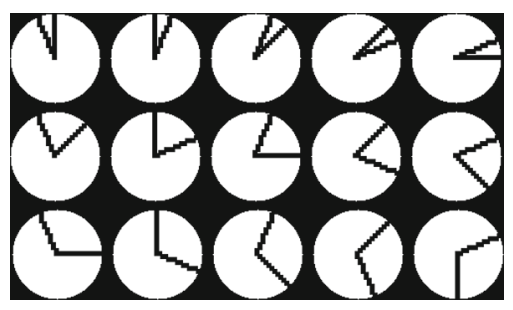

Fig. 1. Optimal stimuli for some V4 neurons that respond selectively to angles and curves, which can be characterized by two orientations such as two arms of an angle (redrawn from [38]).

while others are sensitive to a combination of adjacent contour elements. Moreover, the experiments of the referred authors reveal that on average V4 neurons have stronger responses to convex features rather than to concave ones which is consistent with the perceptual dominance of convexity found in psychophysics.

Similar to experimental neurophysiological studies, there is not much work on computational modelling of V4. A computational model of V4 neurons has been proposed in [44-46]. The response of a V4-like unit introduced in the referred papers depends on the Euclidean distance between a new input and a stored prototype where both input and prototype are local patterns of Gabor (energy) filter-like responses across different orientations and scales. Such a model will respond to an input pattern even if it contains only a part of the prototype. A missing part can, however, radically change a shape. For instance, a pattern that is formed by two line segments that make an angle is perceptually different from a pattern that consists of one of the constituent line segments. An Euclidean-distance model will, however, find these two patterns similar to a considerable extent. Furthermore, Euclidean-distance models are sensitive to the presence of noise or texture and to contrast variations. Those models are not invariant to any geometrical transformations.

There is psychophysical evidence [18] showing that curvatures are likely detected by an AND-type operation, which considers the responses of some afferent sub-units (sensitive for different parts of the curve pattern) and combines them by multiplication. This is in contrast to Euclidean-distance models that inherently involve addition. An AND-type model is activated only when it receives stimulation from all its afferent input, i.e. all contour parts that form a curve pattern are present. It will not respond when any of its inputs are not stimulated, i.e. any of the constituent parts of a curve pattern is absent. In the following, we propose and use such an AND-type model.

Fidler and Leonardis [16] propose to combine Gabor filter responses for vertex detection. They use local statistical analysis to identify two dominant orientations around a given point and use the corresponding channels in a bank of Gabor filters to detect vertices. This type of operator resembles the properties of shape-selective V4 neurons. At a next level they combine the responses of such operators in a similar way in order to define detectors of more complex 
contour features that resemble the properties of some TEO neurons. Their approach is also vulnerable to contrast, noise and texture, and is also not robust to geometrical transformations.

In the following, we propose nonlinear filters that can detect lines, vertices and more complex contour features, similar to some V4 neurons. We call these filters COSFIRE (Combination of Shifted Filter Responses). The response of such a COSFIRE filter is assembled from selected responses of orientationselective filters. We configure such a filter by selecting given channels of a bank of orientation-selective filters and combining their responses by a weighted geometric mean. The selection of channels is determined by the local pattern that needs to be detected. This pattern is specified by the user as an area of interest in a training image. The COSFIRE filters configured with given local patterns can successfully detect the same and similar patterns in test images. The degree of similarity/generalisation can be controlled by changing the values of certain model parameters. We show how a COSFIRE filter achieves rotation-, scale- and reflection-invariance.

The rest of this paper is organized as follows: In Sect. 2 we explain how a COSFIRE filter can be configured by a specified prototype pattern of interest. In Sect. 3, we demonstrate the effectiveness of the proposed COSFIRE filters by applying them to three practical applications: the detection of vascular bifurcations in retinal fundus images, the detection and recognition of traffic signs in complex scenes, and the recognition of handwritten digits. Section 4 contains a discussion of some aspects of the proposed trainable approach and finally we draw our conclusions in Sect. 5 .

\section{Computational Model and Its Implementation}

A COSFIRE filter takes as input the responses of a collection of orientationselective filters that model V1/V2 cells. Here we use Gabor filters as they have been widely used for more than two decades. Other orientation-selective filters, such as CORF [4,5], may also be used. A COSFIRE filter response is then computed as the weighted geometric mean of the responses of certain Gabor filters at specific locations with respect to its receptive field center. The type (orientation-selectivity and scale) of Gabor filters and the relative locations at which we combine their responses are determined in an automatic configuration process which we explain below.

\subsection{Afferent Inputs: Gabor Filters}

We denote by $\left|g_{\lambda, \theta}(x, y)\right|_{t_{1}}$ the thresholded response of a Gabor filter with orientation preference of $\theta$ and a spatial wavelength preference of $\lambda$ to an input image. Such a filter is described by other parameters, namely bandwidth, spatial aspect ratio, and phase offset, which we set as suggested in [41]. We normalize each Gabor function that we use in such a way that the total sums of all the positive and negative values are 1 and -1 , respectively. This normalization ensures 
that the response to a line of width $w$ will be largest for a symmetrical filter of preferred wavelength $\lambda=2 w$. It also ensures that the response to an image of constant intensity is 0 . Without such normalization, this is true only for antisymmetrical filters.

\subsection{Configuration}

A COSFIRE filter is configured by an automatic procedure that analyses the contour properties of a given local pattern, that we call a prototype. This is achieved in a single-step training phase where the user specifies a point of interest and a bounding box that surrounds a prototype of interest in a training image. Fig. 2a shows an input image with an enframed vertex that is considered as a prototype pattern.

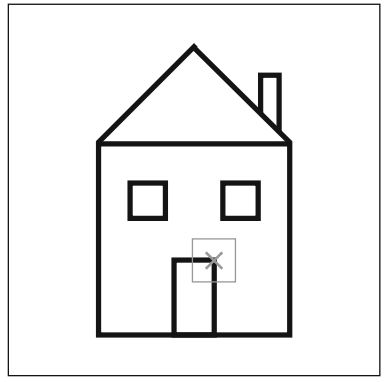

(a)

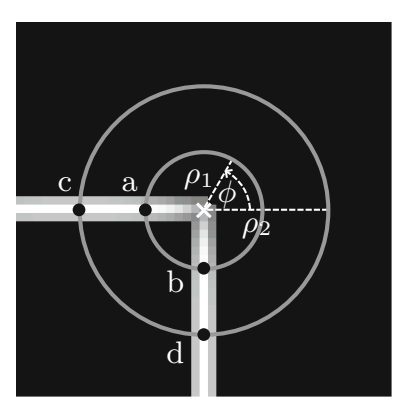

(b)

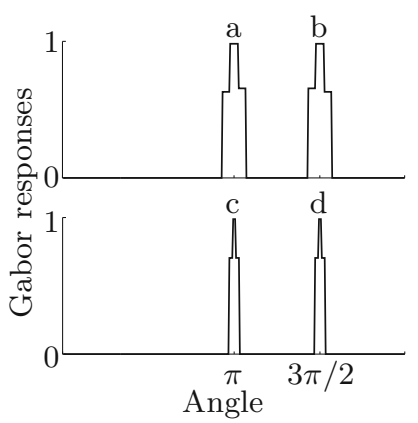

(c)

Fig. 2. (a) Training image of size $284 \times 284$ pixels. The cross marker and the gray bounding box around it indicate the specified local pattern of interest referred to as prototype. (b) The intensity pixels represent the superimposed thresholded responses $\left(t_{1}=0.4\right)$ of a bank of Gabor filters with eight orientations $\left(\theta \in\left\{0, \frac{\pi}{8}, \ldots, \frac{7 \pi}{8}\right\}\right)$ and five wavelengths $(\lambda \in\{4,4 \sqrt{2}, \ldots, 16\})$ to the vertex prototype specified in (b). The top and bottom plots in (c) illustrate the Gabor responses along the inner and outer concentric circles in (b), respectively. The labels 'a', 'b', 'c', and 'd' indicate the local maxima points and are marked with black dots in (c). The location of each such point $i$ is represented in polar coordinates $\left(\rho_{i}, \phi_{i}\right)$ relative to the specified point of interest.

The automatic analysis consists of three steps. First, we apply a bank of Gabor filters to the training image that contains the selected prototype, and threshold their responses using $t_{1}$, Fig. 2b. Here we use symmetric Gabor filters as the prototype is characterized by bar structures. Second, we consider the superimposed Gabor (thresholded) responses on the point of interest and along a number of $k$ concentric circles (here $k=2$ ) around that point. Then, we choose the locations along the concentric circles at which we achieve local maxima Gabor responses in an arc neighbourhood of $\pi / 8$, Fig. 2c. These locations are converted to polar coordinates $(\rho, \phi)$ with respect to the given point of interest. They mark 
the positions of the dominant contours in the prototype. For each such a location we determine the channels $(\lambda, \theta)$ of the Gabor filters that exceed a fraction $t_{2}$ (here $t_{2}=0.5$ ) of the maximum response at that position. Third, we denote by $S_{f}$ a set of 4-tuples that represents the channels and the respective locations of the Gabor filters that satisfy the above criteria for a prototype feature $f$ :

$$
S_{f}=\left\{\left(\lambda_{i}, \theta_{i}, \rho_{i}, \phi_{i}\right) \mid i=1 \ldots n\right\}
$$

where $n$ is the number of involved Gabor filters. Each tuple $\left(\lambda_{i}, \theta_{i}, \rho_{i}, \phi_{i}\right)$ represents the characteristics of a contour part in the pattern of interest.

\subsection{COSFIRE Filter Response}

For each tuple $\left(\lambda_{i}, \theta_{i}, \rho_{i}, \phi_{i}\right)$ in set $S_{f}$ we apply the Gabor filter with wavelength $\lambda_{i}$ and orientation $\theta_{i}$. Then we consider the Gabor responses in locations defined by the corresponding polar coordinates $\left(\rho_{i}, \phi_{i}\right)$. This is achieved by shifting the Gabor responses by $\rho_{i}$ pixels in the direction opposite to $\phi_{i}$. In this way the Gabor responses of interest meet at the same place, the one that we consider the support (or receptive field) center of the concerned COSFIRE filter.

Before shifting, however, we apply a blurring function to the Gabor responses in order to achieve some tolerance with respect to the preferred positions. For blurring we use a Gaussian function $G_{\sigma}(x, y)$ centered on the preferred position and compute the maximum of the weighted Gabor responses. The considered neighbours are determined by a standard deviation $\sigma=\sigma_{0}+\alpha \rho_{i}$ that grows linearly with the distance $\rho_{i}$ from the support center of the COSFIRE filter at hand. The positive values of parameters $\sigma_{0}$ and $\alpha$ are constants. The value of $\sigma_{0}$ is the standard deviation used at the support center of the concerned COSFIRE filter and the value $\alpha$ determines the extent of tolerance: tolerance increases with an increasing value of $\alpha$. We denote by $s_{\lambda_{i}, \theta_{i}, \rho_{i}, \phi_{i}}(x, y)$ the blurred and shifted Gabor response for tuple $\left(\lambda_{i}, \theta_{i}, \rho_{i}, \phi_{i}\right)$ in set $S_{f}$, and denote by $r_{S_{f}}(x, y)$ the response of a COSFIRE filter:

$$
r_{s_{f}}(x, y)=\left(\prod_{i=1}^{n} s_{\lambda_{i}, \theta_{i}, \rho_{i}, \phi_{i}}(x, y)^{\omega_{i}}\right)^{1 / \sum_{i=1}^{n} \omega_{i}}
$$

where $\omega_{i}=\exp ^{-\frac{\rho_{i}^{2}}{2 \sigma^{\prime 2}}}$. Here we use $\sigma^{\prime}=\left(-\rho_{\max }^{2} / 2 \log 0.5\right)^{\frac{1}{2}}$ where $\rho_{\max }=$ $\max _{i \in\left\{1 \ldots\left|S_{f}\right|\right\}} \rho_{i}$. With such a weighting scheme the weights in the center $(\rho=0)$ have a maximum value $\omega=1$, and the farthest points $\left(\rho=\rho_{\max }\right)$ have a minimum value $\omega=0.5$.

Figure $3 a$ illustrates the detected features in the input image shown in Fig. 2a. The circles surround the local maxima points in the COSFIRE response image that is obtained with Eq. 2.

\subsection{Achieving Invariance to Geometric Transformations}

We achieve invariance to rotation, scale and reflection by simply controlling some parameter values, instead of configuring COSFIRE filters by prototypes that are rotated, scaled or reflected versions of each other. 


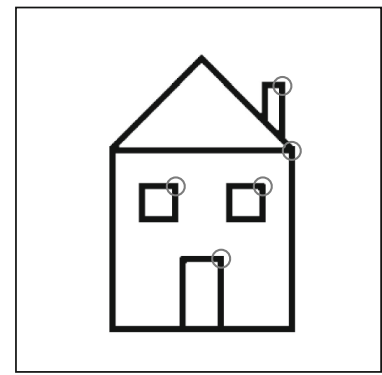

(a)

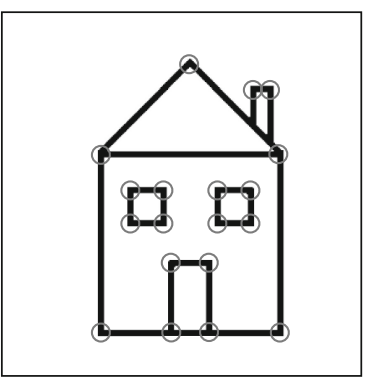

(b)

Fig. 3. Detection of features by a corner-selective COSFIRE filter which is applied in (a) a non-invariant mode and in (b) a rotation-invariant mode. The circles indicate the detected features.

We form a new set $\Re_{\psi}\left(S_{f}\right)=\left\{\left(\lambda_{i}, \theta_{i}+\psi, \rho_{i}, \phi_{i}+\psi\right) \mid \forall\left(\lambda_{i}, \theta_{i}, \rho_{i}, \phi_{i}\right) \in\right.$ $\left.S_{f}\right\}$ by adding an offset $\psi$ to the values of parameters $\theta_{i}$ and $\phi_{i}$. The resulting set represents a COSFIRE filter that is selective for the same prototype $f$ rotated by an angle $\psi$. Rotation invariance is then achieved by considering a set $\Psi$ of equidistant $\psi$ values and then taking the maximum response: $\hat{r}_{S_{f}}=\max _{\psi \in \Psi}\left\{r_{\Re_{\psi}}(x, y)\right\}$. Figure $3 \mathrm{~b}$ illustrates the detection of all right-angled corners irrespective of their orientation $(\Psi=\{0, \pi / 4, \ldots, 7 \pi / 8\})$.

Similarly, we form a new set $T_{v}\left(S_{f}\right)=\left\{\left(v \lambda_{i}, \theta_{i}, v \rho_{i}, \phi_{i}\right) \mid \forall\left(\lambda_{i}, \theta_{i}, \rho_{i}, \phi_{i}\right) \in\right.$ $\left.S_{f}\right\}$ by multiplying with a factor $v$ the values of parameters $\lambda_{i}$ and $\rho_{i}$. This results in a COSFIRE filter that responds to the prototype $f$ scaled by a factor $v$. A scale-invariant response is achieved by considering a set $\Upsilon$ of $v$ values equidistant on a logarithmic scale and then take the maximum response: $\tilde{r}_{S_{f}}(x, y)=\max _{v \in \Upsilon}\left\{r_{T_{v}\left(S_{f}\right)}(x, y)\right\}$.

A new set $\dot{S}_{f}=\left\{\left(\lambda_{i}, \pi-\theta_{i}, \rho_{i}, \pi-\phi_{i}\right) \mid \forall\left(\lambda_{i}, \theta_{i}, \rho_{i}, \phi_{i}\right) \in S_{f}\right\}$ results in a COSFIRE filter that is selective to the prototype $f$ reflected about the $y$-axis. A reflection-invariant response is then computed as $\dot{r}_{S_{f}}(x, y)=\max \left\{r_{S_{f}}, r_{S_{f}}\right\}$.

Finally, we denote by $\bar{r}_{S_{f}}$ the combined rotation-, scale- and reflection-invariant response by taking the maximum value of the rotation and scale-invariant responses of the filters $S_{f}$ and $\dot{S}_{f}: \bar{r}_{S_{f}}(x, y)=\max \left\{\hat{r}_{\Re_{\psi}\left(T_{v}\left(S_{f}\right)\right)}(x, y), \hat{r}_{\Re_{\psi}\left(T_{v}\left(\dot{S}_{f}\right)\right)}(x, y)\right\}$.

\section{Experiments}

In [8] we demonstrated the effectiveness of the COSFIRE filters in three applications: the detection of vascular bifurcations in segmented retinal fundus images, detection and recognition of traffic signs embedded in images of complex scenes, as well as the recognition of handwritten digits, Fig. 4.

Retinal image analysis is gaining popularity as it gives the opportunity to take a non-invasive look at the cardiovascular system of human beings. One important step in this analysis is the detection of vascular bifurcations in the vessel tree. In [7] we evaluated our method, for the detection of vascular bifurcations in 


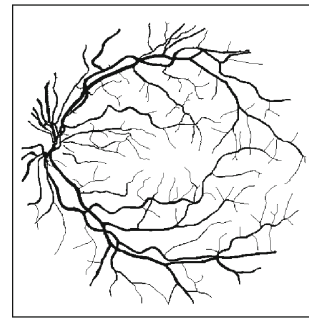

(a)

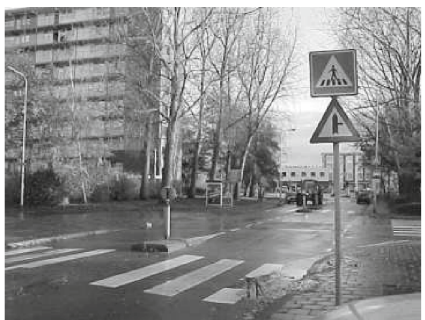

(b)

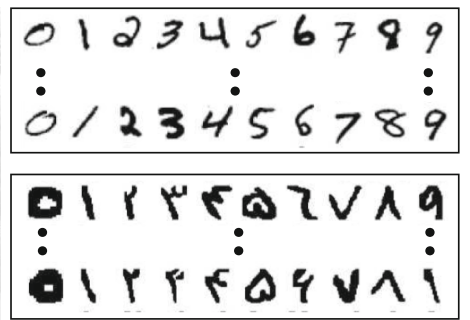

(c)

Fig. 4. Trainable COSFIRE filters are effectively applied to three applications: (a) detection of vascular bifurcations in retinal fundus images, (b) detection and recognition of traffic signs in complex scenes and (c) recognition of (top) western Arabic and (bottom) Farsi handwritten digits.

retinal images, on two benchmark data sets and attained the following results: a precision of $96.94 \%$ at a recall of $97.88 \%$ on 40 images provided in the DRIVE data set [48] and a precision of $96.04 \%$ at a recall of $97.32 \%$ on 20 images taken from the STARE data set [25]. In these experiments, we first configured six COSFIRE filters in a training phase by using different prototypical bifurcations. Then we applied the configured filters to test retinal images in rotation-, scaleand reflection-invariant mode.

In order to show the versatility and robustness of the COSFIRE approach in [8] we performed experiments on a public data set ${ }^{1}$ of outdoor scenes for the detection and recognition of three different traffic signs. This data set was originally published in [20]. We configured three COSFIRE filters to be selective for the three concerned prototypical traffic signs. For a data set of 48 images we were able to localize and recognize all the traffic signs in the data set.

We also demonstrated that the collective responses of a group of COSFIRE filters can also be used to form a shape descriptor. In [6] we applied this shape descriptor to the recognition of handwritten digits, an application that has been extensively used for the evaluation of shape descriptors. We achieved a recognition rate of $99.52 \%$ on the MNIST data set [34] of 70,000 (60,000 training and 10,000 test) western Arabic digits. This result is comparable to the best results ever achieved by other state-of-the-art methods. Furthermore, we achieved a recognition rate of $99.33 \%$ on a data set [31] of 80,000 (60,000 training and 20,000 test) Farsi digits, which is the highest recognition rate ever reported for this data set.

The shape descriptor that we propose is inspired by the neurophysiological concept of population coding. There is evidence [40] that the responses of multiple shape-selective neurons in area V4 of visual cortex can be effectively used as a signature to discriminate between complex shapes.

\footnotetext{
${ }^{1}$ Traffic sign data set is online: http://www.cs.rug.nl/imaging/databases/traffic_sign database/traffic_sign_database.html.
} 


\section{Discussion}

We propose a trainable COSFIRE approach to visual pattern recognition that is inspired by the shape selectivity of V4 neurons in visual cortex. In [8] we demonstrated that the response of COSFIRE filters to test stimuli used in electrophysiological measurements is similar to the response of some V4 neurons.

We demonstrated the effectiveness of the proposed filters in three practical applications: the detection of vascular bifurcations in retinal fundus images, the detection and recognition of traffic signs in outdoor scenes, and the recognition of handwritten digits.

The COSFIRE filters that we propose are trainable, in that the specific feature to which such a filter optimally responds is used to determine the structure of the filter. In a single-step training process, the user specifies a pattern of interest and that pattern is used to configure a corresponding COSFIRE filter. This selectivity is not achieved by template matching, but rather by the determination of the dominant orientations in the concerned pattern and their mutual geometrical arrangement. The proposed filters are highly nonlinear, in that such a filter will only respond when all parts of the concerned feature are present.

Although a COSFIRE filter has a preferred selectivity to the pattern that was used for its configuration, the filter also responds to similar patterns which differ - to a certain extent - in the orientations of the involved lines and edges. The degree of generalization is flexible and can be tuned to the specific needs of the user by proper selection of the filter parameters, We also demonstrate how these filters can be augmented with rotation, scale and refection invariance by simply manipulating some model parameters.

COSFIRE filters are conceptually simple and easy to implement: the filter output is computed as the (weighted) geometric mean of blurred and shifted responses of orientation-selective filters ${ }^{2}$.

\section{Conclusions}

The trainable COSFIRE approach reviewed in this paper is a contribution to the understanding of the visual system of the brain. It shows how information from computational models of the responses of V1/V2 simple and complex cells, can be assembled at the next, V4 level, in order to construct detectors of more complex stimuli, such as angles, curves, line bifurcations and, more generally, local combinations of line and curve segments. We also demonstrated how this understanding of the visual system of the brain can be used to design effective computer vision algorithms.

\section{References}

1. Adelson, E.H., Bergen, J.R.: Spatiotemporal energy models for the perception of motion. J. Opt. Soc. Am. a-Optics Image Sci. Vis. 2(2), 284-299 (1985)

\footnotetext{
${ }^{2}$ The Matlab and $\mathrm{C}++/$ OpenCV implementations of COSFIRE can be downloaded from http://matlabserver.cs.rug.nl.
} 
2. Albrecht, D.G., De Valois, R.L., Thorell, L.G.: Visual cortical-neurons - are bars or gratings the optimal stimuli. Science 207(4426), 88-90 (1980)

3. Andrews, B.W., Pollen, D.A.: Relationship between spatial-frequency selectivity and receptive-field profile of simple cells. J. Physiol. Lond. 287, 163-176 (1979)

4. Azzopardi, G., Petkov, N.: A CORF computational model of a simple cell that relies on LGN input outperforms the Gabor function model. Biol. Cybern. 106(3), 177-189 (2012)

5. Azzopardi, G., Petkov, N.: Contour detection by CORF operator. In: Villa, A.E.P., Duch, W., Érdi, P., Masulli, F., Palm, G. (eds.) ICANN 2012, Part I. LNCS, vol. 7552, pp. 395-402. Springer, Heidelberg (2012)

6. Azzopardi, G., Petkov, N.: A shape descriptor based on trainable COSFIRE filters for the recognition of handwritten digits. In: Wilson, R., Hancock, E., Bors, A., Smith, W. (eds.) CAIP 2013, Part II. LNCS, vol. 8048, pp. 9-16. Springer, Heidelberg (2013)

7. Azzopardi, G., Petkov, N.: Automatic detection of vascular bifurcations in segmented retinal images using trainable COSFIRE filters. Pattern Recogn. Lett. 34(8), 922-933 (2013)

8. Azzopardi, G., Petkov, N.: Trainable COSFIRE filters for keypoint detection and pattern recognition. IEEE Trans. Pattern Anal. Mach. Intell. 35(2), 490-503 (2013)

9. Bovik, A.C.: Analysis of multichannel narrow-band-filters for image texture segmentation. IEEE Trans. Signal Process. 39(9), 2025-2043 (1991)

10. Cristobal, G., Navarro, R.: Space and frequency variant image-enhancement based on a gabor representation. Pattern Recogn. Lett. 15(3), 273-277 (1994)

11. Daugman, J.G.: Uncertainty relation for resolution in space, spatial-frequency, and orientation optimized by two-dimensional visual cortical filters. J. Opt. Soc. Am. a-Optics Image Sci. Vis. 2(7), 1160-1169 (1985)

12. Daugman, J.G.: Complete discrete 2-d gabor transforms by neural networks for image-analysis and compression. IEEE Trans. Acoust. Speech Signal Process. 36(7), 1169-1179 (1988)

13. Daugman, J.G.: High confidence visual recognition of persons by a test of statistical independence. IEEE Trans. Pattern Anal. Mach. Intell. 15(11), 1148-1161 (1993)

14. De Valois, K.K., De Valois, R.L., Yund, E.W.: Responses of striate cortex cells to grating and checkerboard patterns. J. Physiol. (Lond.) 291, 483-505 (1979)

15. De Valois, R.L., Albrecht, D.G., Thorell, L.G.: Cortical cells: bar and edge detectors, or spatial frequency filters? In: S.J. Cool and III Smith, E. L. (eds.) Frontiers in Visual Science, pp. 544-56. Springer, Berlin (1978): Frontiers in Visual Science, Houston, TX, USA, March 1977

16. Fidler, S., Leonardis, A.: Towards scalable representations of object categories: Learning a hierarchy of parts. In: 2007 IEEE Conference on Computer Vision and Pattern Recognition, vol. 1-8, pp. 2295-2302 (2007)

17. Fogel, I., Sagi, D.: Gabor filters as texture discriminator. Biol. Cybern. 61(2), 103-113 (1989)

18. Gheorghiu, E., Kingdom, F.A.A.: Multiplication in curvature processing. J. Vis. 9(2), 1-7 (2009)

19. Goodale, M.A., Milner, A.D.: Separate visual pathways for perception and action. Trends Neurosci. 15(1), 20-25 (1992)

20. Grigorescu, C., Petkov, N.: Distance sets for shape filters and shape recognition. IEEE Trans. Image Process. 12(10), 1274-1286 (2003)

21. Grigorescu, C., Petkov, N., Westenberg, M.A.: Contour detection based on nonclassical receptive field inhibition. IEEE Trans. Image Process. 12(7), 729-739 (2003) 
22. Grigorescu, C., Petkov, N., Westenberg, M.A.: Contour and boundary detection improved by surround suppression of texture edges. Image Vis. Comput. 22(8), 609-622 (2004)

23. Grigorescu, S.E., Petkov, N., Kruizinga, P.: Comparison of texture features based on gabor filters. IEEE Trans. Image Process. 11(10), 1160-1167 (2002)

24. Hanazawa, A., Komatsu, H.: Influence of the direction of elemental luminance gradients on the responses of v4 cells to textured surfaces. J. Neurosci. 21(12), 4490-4497 (2001)

25. Hoover, A., Kouznetsova, V., Goldbaum, M.: Locating blood vessels in retinal images by piecewise threshold probing of a matched filter response. IEEE Trans. Med. Imaging 19(3), 203-210 (2000)

26. Hubel, D.H.: Exploration of the primary visual-cortex, 1955-78. Nature 299(5883), 515-524 (1982)

27. Hubel, D.H., Wiesel, T.N.: Receptive fields, binocular interaction and functional architecture in cats visual cortex. J. Physiol. (Lond.) 160(1), 106-154 (1962)

28. Hubel, D.H., Wiesel, T.N.: Sequence regularity and geometry of orientation columns in monkey striate cortex. J. Comp. Neurol. 158(3), 267-294 (1974)

29. Jain, A.K., Farrokhnia, F.: Unsupervised texture segmentation using gabor filters. Pattern Recogn. 24(12), 1167-1186 (1991)

30. Jones, J.P., Palmer, L.A.: An evaluation of the two-dimensional gabor filter model of simple receptive-fields in cat striate cortex. J. Neurophysiol. 58(6), 1233-1258 (1987)

31. Khosravi, H., Kabir, E.: Introducing a very large dataset of handwritten Farsi digits and a study on their varieties. Pattern Recogn. Lett. 28(10), 1133-1141 (2007)

32. Kovesi, P.: Image features from phase congruency. Videre 1(3), 1-27 (1999)

33. Kulikowski, J.J., Bishop, P.O.: Fourier-analysis and spatial representation in the visual-cortex. Experientia 37(2), 160-163 (1981)

34. LeCun, Y., Huang, F.J., Bottou, L.: Learning methods for generic object recognition with invariance to pose and lighting. In: Proceedings of the 2004 IEEE Computer Society Conference on Computer Vision and Pattern Recognition, vol 2, pp. 97-104 (2004)

35. Macleod, I.D.G., Rosenfeld, A.: Visibility of gratings - spatial frequency channels or bar-detecting units. Vision. Res. 14(10), 909-915 (1974)

36. Manjunath, B.S., Shekhar, C., Chellappa, R.: A new approach to image feature detection with applications. Pattern Recogn. 29(4), 627-640 (1996)

37. Mehrotra, R., Namuduri, K.R., Ranganathan, N.: Gabor filter-based edgedetection. Pattern Recogn. 25(12), 1479-1494 (1992)

38. Pasupathy, A., Connor, C.E.: Responses to contour features in macaque area v4. J. Neurophysiol. 82(5), 2490-2502 (1999)

39. Pasupathy, A., Connor, C.E.: Shape representation in area v4: Position-specific tuning for boundary conformation. J. Neurophysiol. 86(5), 2505-2519 (2001)

40. Pasupathy, A., Connor, C.E.: Population coding of shape in area v4. Nat. Neurosci. 5(12), 1332-1338 (2002)

41. Petkov, N.: Biologically motivated computationally intensive approaches to image pattern-recognition. Future Gener. Comput. Syst. 11(4-5), 451-465 (1995)

42. Petkov, N., Subramanian, E.: Motion detection, noise reduction, texture suppression, and contour enhancement by spatiotemporal gabor filters with surround inhibition. Biol. Cybern. 97(5-6), 423-439 (2007)

43. Petkov, N., Westenberg, M.A.: Suppression of contour perception by band-limited noise and its relation to non-classical receptive field inhibition. Biol. Cybern. 88(10), 236-246 (2003) 
44. Riesenhuber, M., Poggio, T.: Hierarchical models of object recognition in cortex. Nat. Neurosci. 2(11), 1019-1025 (1999)

45. Serre, T., Kouh, M., Cadieu, C., Knoblich, U., Kreiman, G., Poggio, T.: A theory of object recognition: computations and circuits in the feedforward path of the ventral stream in primate visual cortex. AI Memo 2005-036/CBCL Memo 259, Massachusetts Inst. of Technology, Cambridge (2005)

46. Serre, T., Wolf, L., Bileschi, S., Riesenhuber, M., Poggio, T.: Robust object recognition with cortex-like mechanisms. IEEE Trans. Pattern Anal. Mach. Intell. 29(3), 411-426 (2007)

47. Shapley, R., Caelli, T., Morgan, M., Rentschler, I.: Computational theories of visual perception. In: Spillmann, L., Werner, J.S. (eds.) Visual Perception: The Neurophysiological Foundations, pp. 417-448. Academic, New York (1990)

48. Staal, J., Abramoff, M.D., Niemeijer, M., Viergever, M.A., van Ginneken, B.: Ridge-based vessel segmentation in color images of the retina. IEEE Trans. Med. Imaging 23(4), 501-509 (2004)

49. Tan, T.N.: Texture edge-detection by modeling visual cortical channels. Pattern Recogn. 28(9), 1283-1298 (1995)

50. Turner, M.R.: Texture-discrimination by gabor functions. Biol. Cybern. 55(2-3), 71-82 (1986)

51. Tyler, C.W.: Selectivity for spatial-frequency and bar width in cat visual-cortex. Vis. Res. 18(1), 121-122 (1978)

52. Ungerleider, L.G., Mishkin, M.: Two Cortical Visual Systems. MIT Press, Cambridge (1982)

53. Von Der Heydt, R.: Approaches to visual cortical function. Rev. Physiol. Biochem. Pharmacol. 108, 69-150 (1987)

54. Wu, P., Manjunath, B.S., Newsam, S., Shin, H.D.: A texture descriptor for browsing and similarity retrieval. Signal Process.-Image Commun. 16(1-2), 33-43 (2000)

55. Zeki, S.M.: Color coding in rhesus-monkey prestriate cortex. Brain Res. 53(2), 422-427 (1973) 